\title{
INFLUENCE OF HEAT TREATMENT CONDITION ON PHOTOCATALYTIC PROPERTIES OF OXIDIZED TIN FILM
}

\author{
DAISUKE YONEKURA \\ Department of Mechanical Engineering, The University of Tokushima, 2-1 Minami-josanjima \\ Tokushima, Tokushima 770-8506, Japan \\ yonekura@me.tokushima-u.ac.jp \\ SHUHEI KATSURA \\ School of Mechanical Engineering, The University of Tokushima, 2-1 Minami-josanjima \\ Tokushima, Tokushima 770-8506, Japan \\ RI-ICHI MURAKAMI \\ Department of Mechanical Engineering, The University of Tokushima, 2-1 Minami-josanjima \\ Tokushima, Tokushima 770-8506, Japan \\ murakami@me.tokushima-u.ac.jp
}

\begin{abstract}
Nitride doped titanium oxide $\left(\mathrm{TiO}_{\mathrm{x}} \mathrm{N}_{\mathrm{y}}\right)$ is a photocatalytic material which acts under visible light, while conventional titanium oxide acts only in ultra violet light. In this study, we tried to make nitride doped titanium film by oxidizing of TiN film. The TiN films were deposited on the quartz substrate by DC magnetron sputtering. The film structure was analyzed by X-ray diffraction. The photocatalytic decomposing rate was measured using methylene blue solution under black light and fluorescent lamp. As a result, post annealing process after oxidizing process is unsuitable to realize a high photocatalytic activity for oxidized TiN. On the other hand, the samples oxidized in nitrogen rich atmosphere showed the obvious photocatalytic activity under visible light irradiation. This reason is that the oxidation of the film surface is controlled and nitrogen atom left in film surface by nitrogen gas supplying. In conclusion, oxidizing of TiN film in nitrogen rich atmosphere is suitable to realize a high photocatalytic activity.
\end{abstract}

Keywords: $\mathrm{TiN}$ film, $\mathrm{TiO}_{\mathrm{x}} \mathrm{N}_{y}$, oxidation, photocatalytic activity, sputtering.

\section{Introduction}

Titanium dioxide $\left(\mathrm{TiO}_{2}\right)$ is well known as a photocatalytic material. However, $\mathrm{TiO}_{2}$ only reacts under irradiation with ultraviolet (UV) light $(\sim 380 \mathrm{~nm})$. To achieve photocatalytic activity under visible light irradiation, doping of impurities, in particular anionic species, is effective. ${ }^{1-3}$ Nitride doped titanium oxide $\left(\mathrm{TiO}_{\mathrm{x}} \mathrm{N}_{\mathrm{y}}\right)$ is a photocatalytic material which reacts under visible light irradiation. Many researchers have reported the synthesis methods and the photocatalytic activity. Physical vapor deposition (PVD) is one of the useful methods to synthesize the $\mathrm{TiO}_{\mathrm{x}} \mathrm{N}_{\mathrm{y}}$ film directly on a substrate. ${ }^{4-6}$ 
Oxidizing treatment of titanium nitride (TiN) film is one of the favorable methods to produce $\mathrm{TiO}_{\mathrm{x}} \mathrm{N}_{\mathrm{y}}$ thin film. TiN film is generally deposited by the PVD method and it is widely used to improve the wear resistance of tools due to its high hardness. TiN can be oxidized above about $673 \mathrm{~K}$ in air $^{7}$ with remaining nitrogen atoms in the crystal. ${ }^{8}$ In our previous study, we investigated the influence of oxidizing temperature and pressure on the photocatalytic properties of oxidized TiN under irradiation of UV and visible light using TiN powder and TiN film. ${ }^{9}$ As a result, obvious photocatalytic activity was obtained under visible light irradiation for oxidized TiN powder. However, the photocatalytic activity of oxidized TiN films was low under visible light compared with oxidized TiN powder. The low photocatalytic activity for oxidized TiN film might be caused by excessive substitution of oxygen atoms for nitrogen atoms. To reduce the influence of excessive substitution, two approaches will be effective; one is diffusion of nitrogen atoms by post annealing, another is prevention of excessive oxidation by oxidizing in nitrogen rich atmosphere.

In this study, TiN films were deposited on quartz substrates using DC magnetron sputtering and we examined the influence of post annealing and oxidizing in nitrogen rich atmosphere on the photocatalytic properties of oxidized TiN under UV and visible light irradiation.

\section{Experimental Procedure}

A quartz plate $(150 \times 50 \times 2 \mathrm{~mm})$ was used as a substrate. Firstly, TiN film was deposited on the substrate using pure Ti target $(99.9 \%)$ in a mixture of $\operatorname{Ar}(99.999 \%)$ and $\mathrm{N}_{2}$ (99.999\%) gas using a DC magnetron sputtering system (Fujikosi, Japan). The substrates were placed on a rotating substrate holder in a vacuum chamber. After the vacuum chamber was evacuated to about $2.5 \times 10^{-3} \mathrm{~Pa}$, a pre-sputtering process was performed for 5 minutes in an Ar atmosphere. Then, TiN films were deposited on the substrates. The deposition conditions were as follows: working pressure, target current, bias voltage, anode voltage, deposition temperature and nitrogen gas flow ratio were $0.9 \mathrm{~Pa}, 2 \mathrm{~A},-30 \mathrm{~V}$, $10 \mathrm{~V}, 573 \mathrm{~K}$ and $20 \%$, respectively. TiN film of $1.0 \mu \mathrm{m}$ thick was deposited on the substrate by controlling the deposition time based on the deposition rate of $1.0 \mu \mathrm{m} / \mathrm{h}$.

For post annealing samples, the TiN films were oxidized at 723, 823 and $923 \mathrm{~K}$ under atmospheric pressure (AP) for 6 hours using a vacuum furnace. After the oxidizing treatment, post annealing process was performed in high vacuum condition for 2 hours at same temperature as oxidizing treatment. For the samples oxidized in nitrogen rich atmosphere, the TiN films were oxidized at 723, 823 and $923 \mathrm{~K}$ under AP and $1.0 \mathrm{kPa}$ absolute pressure for 6 hours using a vacuum furnace. The samples oxidized in nitrogen rich atmosphere were named "NR" series and the samples oxidized in air were named "AIR" series. The nitrogen gas flow rate was controlled at $1.0 \mathrm{~L} / \mathrm{min}$ during the oxidizing process. Film thickness was measured using a stylus surface profilometer $\left(\mathrm{DEKTAK}^{3}\right.$, Veeco Instrument, USA). The structure of the films were analyzed by X-ray diffraction (RINT2000, Rigaku, Japan) with a $\mathrm{Cu} \mathrm{K \alpha}$ source under an applied voltage of $40 \mathrm{kV}$ and 
a current of $20 \mathrm{~mA}$. Depth profile near a film surface was analyzed by SIMS (MiniSIMS, Millbrook, UK).

The photocatalytic activity was evaluated by measuring the decomposition rate of methylene blue solution ( $\mathrm{MB}, \mathrm{C}_{16} \mathrm{H}_{18} \mathrm{~N}_{3} \mathrm{~S}-\mathrm{Cl}-3 \mathrm{H}_{2} \mathrm{O}$ ) under irradiation of $1.0 \mathrm{~mW} / \mathrm{cm}^{2}$ ultraviolet via a black light lamp, and under visible light of 6000 Lux via a fluorescent lamp. The MB solution was poured into an acrylic pipe of $40 \mathrm{~mm}$ bore, which was placed on the sample. Prior to measuring the photocatalytic activity, the samples were dipped into the MB solution for 6 hours in a dark place to equilibrate the adsorption-desorption processes of $\mathrm{MB}$ on the film. Then the photocatalytic activity test was started. The concentration of the MB solution was measured every 20 minutes. The absorption spectrum of the MB solution was measured by spectrometer (V-570, JASCO Corp., Japan) in the wavelength range $200-800 \mathrm{~nm}$. The change of concentration of MB per unit time indicates the photocatalytic activity. Decomposition rate was obtained from the fitting line of the relationship MB concentration and irradiation time by liner least square method. ${ }^{10}$

\section{Experimental Results and Discussion}

\subsection{Effect of post annealing process}

Figure 1 shows the results of the decomposition tests of MB solution under UV and visible light irradiation using AIR series oxidized under AP with/without post annealing process. The decomposition rate was degraded by post annealing process for all temperature, in particular under UV irradiation.
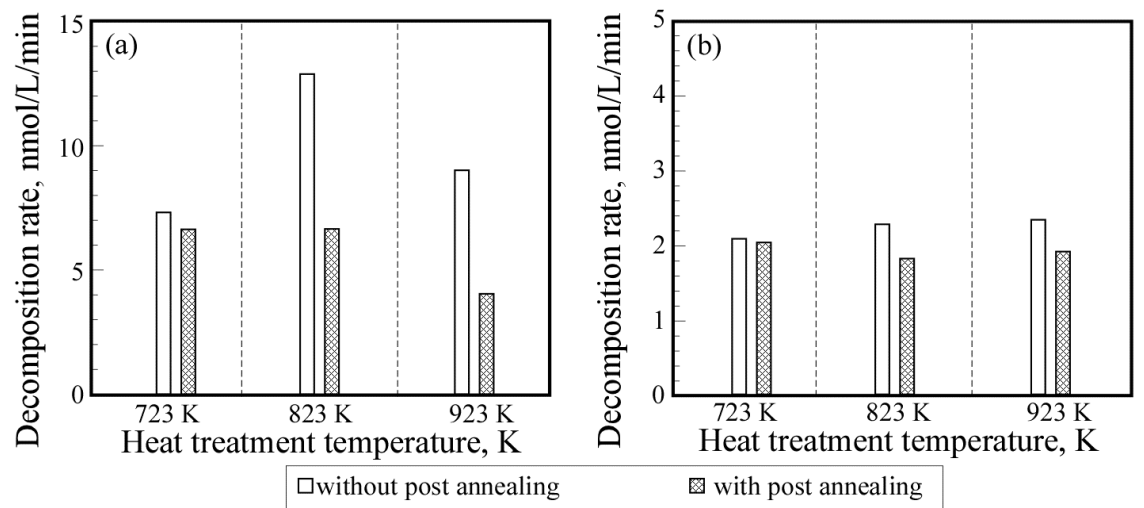

Fig. 1 Decomposition rate of methylene blue solution for AIR series oxidized under atmospheric pressure with/without post annealing process; (a) under UV irradiation, (b) under visible light irradiation.

Figure 2 shows the X-ray diffraction patterns of AIR series oxidized under AP with and without post annealing process. The diffraction peaks corresponding to TiN disappeared for all samples by oxidizing treatment. All films showed a mixture phase of anatase and rutile regardless of with/without post annealing process, however, the diffraction intensity was reduced by the post annealing process for all peaks. Although 
the post annealed samples showed high relative amount of anatase phase compared with without post annealing samples, the photocatalytic activity was degraded by post annealing process as shown in Fig. 1. These results indicate that the crystallinity becomes worse by the post annealing process. The degradation of crystallinity might be caused by generating defects in crystal due to removing nitrogen atoms. Therefore, the post annealing process is unsuitable to realize a high photocatalytic activity.
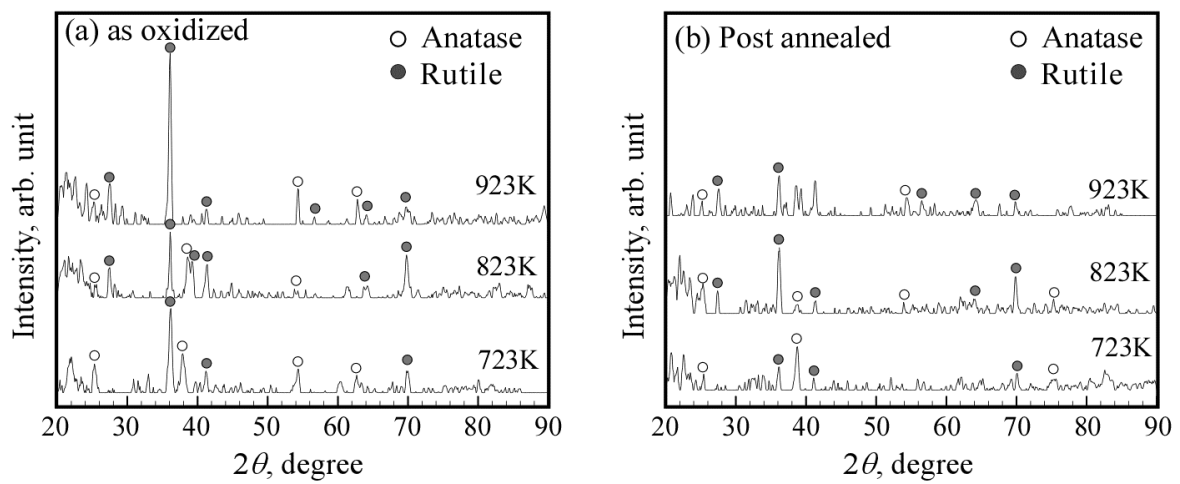

Fig. 2 XRD patterns of films; (a) as oxidized TiN film and (b) post annealed TiN film after oxidizing treatment.

\subsection{Effect of oxidizing in nitrogen rich atmosphere}

Photocatalytic activity was evaluated for NR and AIR series oxidized under AP and $1.0 \mathrm{kPa}$. Figure 3 shows the decomposition rate in irradiation of UV light and visible light irradiation. In the case of the samples oxidized under AP, NR series indicated higher decomposition rate under visible light irradiation (Fig. 3 (b)) compared with AIR series as shown in Fig. 2. For these samples, the decomposition rate increased with increasing the oxidizing temperature and the influence of oxidizing temperature on the photocatalytic activity was similar between NR and AIR series. However, the decomposition rate was decreased or same level under UV irradiation when the samples were oxidized in nitrogen rich atmosphere (Fig. 3 (a)).
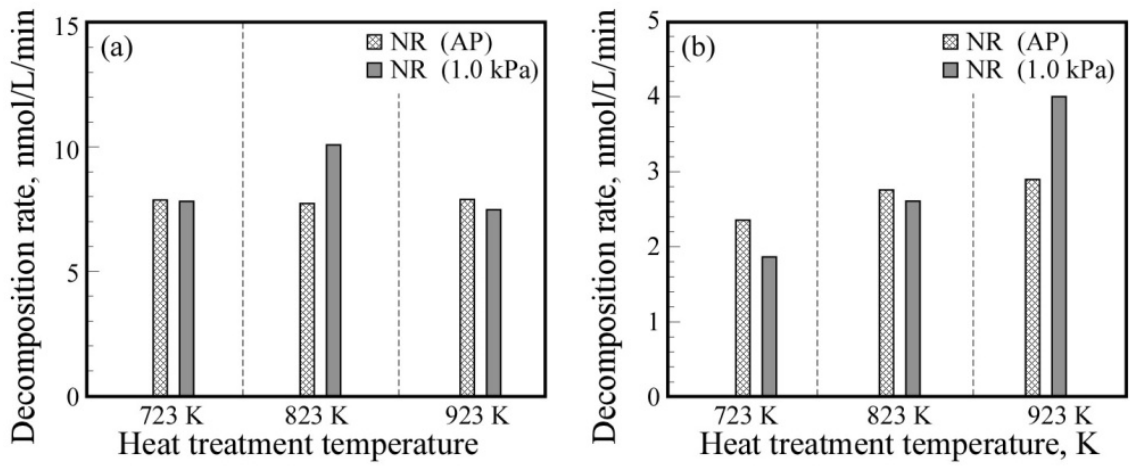

Fig. 3 Decomposition rate of methylene blue solution for oxidized TiN film oxidized under various conditions; (a) under UV irradiation, (b) under visible light irradiation. 
Similar results were obtained for the samples oxidized at $1.0 \mathrm{kPa}$. The effect of oxidizing in nitrogen rich atmosphere clearly appeared under visible light irradiation and the highest decomposition rate of $4.01 \mathrm{nmol} / \mathrm{L} / \mathrm{min}$ was obtained at $923 \mathrm{~K}$ under visible light irradiation.

Figure 4 shows the XRD patterns of the samples. After oxidizing process, the diffraction peaks corresponding to TiN disappeared for all samples. All samples consisted of anatase and rutile phases regardless of processing pressure and supplying nitrogen gas during oxidizing process. The diffraction intensity for NR series were slightly weaker than that of AIR series, however the difference was small. In addition, the XRD patterns also showed no obvious difference for both oxidizing pressures.
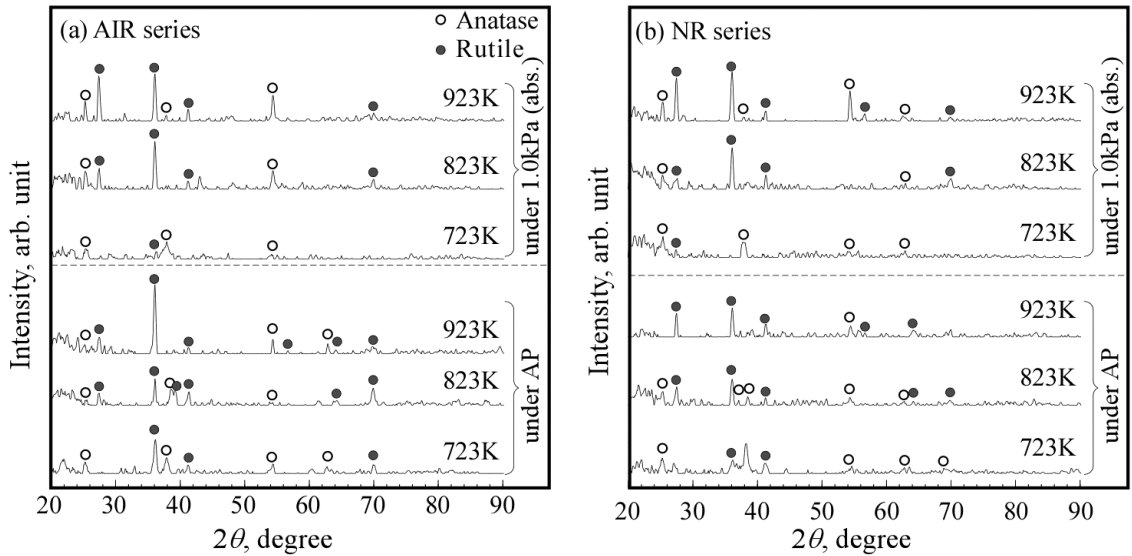

Fig. 4 XRD patterns of oxidized TiN films oxidized under various conditions.

Figure 5 shows the result of depth analysis of oxygen distribution using SIMS for the NR series oxidized at $923 \mathrm{~K}$ under $1.0 \mathrm{kPa}$, which showed the highest photocatalytic activity under visible light irradiation. Figure 5 also shows the result for AIR series oxidized under same condition as a reference. Carbon signal, which is uniformly detected during depth analysis, is used as reference signal and oxygen content in film is shown as signal intensity ratio $\mathrm{O} / \mathrm{C}$.
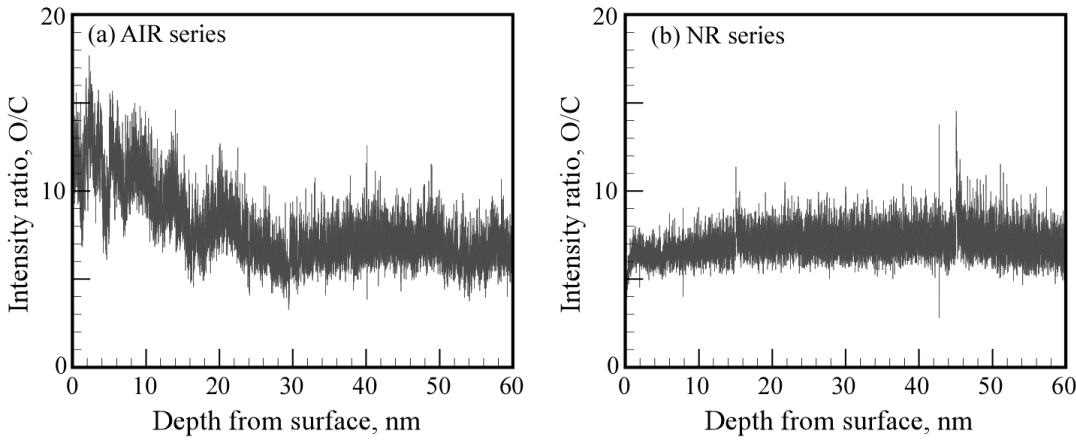

Fig. 5 Result of depth analysis of film surface oxidized at $923 \mathrm{~K}$ under $1.0 \mathrm{kPa}$. 
The signal intensity ratio $\mathrm{O} / \mathrm{C}$ of $\mathrm{NR}$ series was almost constant in all depth range, whereas high $\mathrm{O} / \mathrm{C}$ ratio was obtained near the film surface for AIR series. The result means that oxidizing in nitrogen rich atmosphere is effective to prevent excessive substitution of oxygen atoms for nitrogen atoms and is more effective than oxidizing in air. In conclusion, high photocatalytic activity under visible light irradiation can be achieved by oxidizing at high temperature in vacuum with nitrogen rich atmosphere because the condition prevents excessive oxidation effectively with keeping anatase phase.

\section{Conclusions}

In this study, we examined the influence of post annealing and oxidizing in nitrogen rich atmosphere on the photocatalytic properties of oxidized TiN under UV and visible light irradiation. The results are summarized as follows:

(i) The post annealing process after oxidizing process is unsuitable to realize a high photocatalytic activity.

(ii) Oxidizing of TiN film in nitrogen rich atmosphere under atmospheric pressure improves photocatalytic activity under visible light irradiation regardless of oxidizing temperature.

(iii) Oxidizing at high temperature in nitrogen rich atmosphere, especially in vacuum, is effective to improve photocatalytic activity under visible light irradiation.

\section{References}

1. K. Iino K, M. Kitano, M. Takeuchi, M. Matsuoka. and M. Anpo, Current Applied Physics 6, $982(2006)$.

2. I. Othman, R.M. Mohamed and F.M. Ibrahem, Journal of Photochemistry and Photobiology A: Chemistry 189, 80 (2007).

3. D. Heřman, J. Š́cha and J. Musil, Vacuum 81, 285 (2006).

4. R. Asahi, T. Morikawa, T. Ohwaki, K. Aoki and Y. Taga, Science 293, 269 (2001).

5. M.-S. Wong, H.-P. Chou and T.-S.Yang, Thin Solid Films 494, 244 (2006).

6. S.-W. Park and J.-E. Heo, Separation and Purification Technology 58, 200 (2007).

7. F.-H. Lu and H.-Y. Chen, Thin Solid Films 355-356, 274 (1999).

8. T. Morikawa, R. Asahi, T. Ohwaki., K. Aoki and Y. Taga, Japanese Journal of Applied Physics 40, L561 (2001).

9. D. Yonekura, Y. Harauchi, S. Katsura and R. Murakami, Journal of Solid Mechanics and Materials Engineering 3, 1238 (2009).

10. Wet-type Evolution Method for Performance of Photocatalyst (in Japanese), the Japanese Association of Photocatalyst Products (2004). 